\title{
COMUNICAÇÃO
}

\section{MICRORGANISMOS ASSOCIADOS A FRUTOS DE DIFERENTES CULTIVARES DE NOZ PECAN}

\author{
Microorganisms associated with fruits of different cultivars of pecan nut
}

\author{
Nádia Izumi Terabe ${ }^{1}$, Cristiane Moreno Martins², Martin Homechin ${ }^{3}$
}

\begin{abstract}
RESUMO
O conhecimento do comportamento natural da nogueira Pecan às principais doenças é de suma importância para o estabelecimento do planejamento da implantação da cultura. O controle fitossanitário e tratos culturais devem ser realizados de modo a não comprometerem a qualidade do produto final, as amêndoas. Foram avaliadas nozes produzidas na safra de 2005 e oriundas de Uraí-PR, pelas cultivares Burkett, Frotscher e Moneymaker, para identificar e quantificar os microrganismos associados à amêndoas e cascas dos frutos, bem como observar diferenças entre organismos colonizadores das cultivares. Os frutos foram avaliados na pós-colheita, aos trinta dias de armazenamento em ambiente, através da metodologia do papel de filtro, sendo submetidos ou não à assepsia superficial. $O$ fungo Cladosporium caryigenum, promotor da rancificação das amêndoas foi observado, em amêndoas e cascas, nas cultivares Burkett, Frotscher e Moneymaker; Fusarium sp., foi encontrado em porcentuais elevados, tanto em amêndoas quanto em cascas das três cultivares estudadas; Cephalothecium roseum, causador do mofo róseo em amêndoas, na cultivar Frotscher. Aspergillus sp. e Penicillium sp., causadores de emboloramento e produtores de aflotoxinas foram observados em porcentuais representativos, em amêndoas da cultivar Frotscher e em amêndoas e cascas das cultivares Frotscher, Burkett e Moneymaker, respectivamente. Os maiores porcentuais de perda do rendimento foram observados na cultivar Burkett, por causa da incidência de Colletotrichum sp., causador da antracnose em amêndoas, que acarreta escurecimento e deterioração do produto final, levando-o ao descarte.
\end{abstract}

Termos para indexação: Carya illinoensis, patologia de sementes, sanidade, armazenamento, Cladosporium caryigenum.

\begin{abstract}
The knowledgement of the natural behavior of the main diseases of Pecan nut is of utmost importance for the stablishment of an implantation plan for that culture. The phytosanitary control and cultural treatments should be performed in order not to change the quality of the final product. Nuts harvest in 2005 from Uraí-PR of Burkett, Frotscher and Moneymaker cultivars, were evaluated in order to identify and quantify associated microorganisms in the nuts and fruit cover, as well to observe the differences among the microorganisms that colonize the different cultivars. The fruits were evaluated at post-harvest, thirty days kept in natural temperature, through filter paper method, being submitted or not to surface assepsy. The fungi Cladosporium caryigenum, rancification promoter to the almonds was observed on almonds and covers on the cultivars Burkett, Frotscher and Moneymaker; Fusarium sp., was observed in high concentration, on almonds as well as on the cover of all the studied cultivars; Cephalothecium roseum, responsible for the pink mildew, in almonds in the Frotscher cultivar. Aspergillus sp. and Penicillium sp., responsible for mold and aflatoxins formation, were observed in high amount on almonds of the Frotscher cultivar and in almonds and cover of Frotscher, Burkett and Moneymaker, respectively. The highest percentage of loss in yield was observed on the Burkett cultivar, cause of the incidence of Colletotrichum sp., agent responsible for antracnosis on the almonds which provokes causer darkness and deterioration of final product, leading it to discarding.
\end{abstract}

Index terms: Carya illinoensis, seed pathology, health, storage, Cladosporium caryigenum.

\section{(Recebido em 10 de março de 2006 e aprovado em 11 de abril de 2007)}

A nogueira pecan [Carya illinoensis (Wangenh.) K. Koch], pertencente à família Juglandaceae (REIGER, 2004), é espécie frutífera de clima temperado, cultivada principalmente na região sul do Brasil para a produção comercial de nozes. Seus frutos possuem elevado valor nutricional em proteínas, vitaminas, carboidratos e lipídeos, são antioxidantes, ricos em fibras e gama toco fenol e contém sódio livre (INSTITUTE FOR TROPICAL AND SUBTROPICAL CROPS, 2004). Apresentam propriedades medicinais, sendo indicados no controle da má digestão,

\footnotetext{
'Engenheira Agrônoma - Controle de Processos - Monsanto do Brasil LTDA - Estação Experimental de Uberlândia - Rodovia Br 452 , Km 141 - $38400-000$ Uberlândia, MG - nadia.i.terabe@monsanto.com

${ }^{2}$ Bióloga, Mestre em Agronomia - Departamento de Agronomia - Centro de Ciências Agrárias - Universidade Estadual de Londrina/UEL - Cx. P. 6001 86051-990 - Londrina, PR - crismoreno0702@yahoo.com.br - Bolsista do CNPq

${ }^{3}$ Engenheiro Agrônomo, Doutor, Professor - Departamento de Agronomia - Centro de Ciências Agrárias - Universidade Estadual de Londrina/UEL Cx. P. 6001 - 86051-990 - Londrina, PR - homechin@uel.br
} 
febre, gripe, hepatite, malária e dores estomacais (REIGER, 2004). Seu lenho pode ser empregado na marcenaria (SIMÃO, 1998) e as cascas na confecção de compensados e móveis (REIGER, 2004). Também propriedades alelopáticas e fungitóxicas têm sido relatadas em folhas e frutos em razão da presença do composto juglone (BALL, 2004; REIGER, 2004) e do linalool e óleo essencial (REIGER, 2004).

No Brasil, a pecanicultura tem sido desestimulada em decorrência das poucas pesquisas contemplando a forma de implantação e condução da cultura frente às condições climáticas brasileiras, fatores que predispõem as plantas ao ataque por patógenos, principalmente fungos. Atualmente, seu cultivo se concentra em parte da Região Sudeste, em toda a Região Sul e, sob latitudes menores, nos estados de Minas Gerais e Mato Grosso do Sul. Grande parte dos pomares foram implantados no Brasil a partir de incentivos governamentais nas décadas de 60 e 70 e, atualmente, encontram-se abandonados, com a maioria das árvores apresentando sérios problemas fitossanitários e baixa produção (ORTIZ \& CAMARGO, 2005).

Conhecimentos quanto a resistência natural da nogueira frente às principais doenças, é de suma importância ainda na fase de planejamento de sua implantação, podendo representar redução de gastos com tratos culturais. Produtos recomendados e medidas para o controle das doenças da nogueira pecan no Brasil, praticamente não existem, situação que exige conhecimentos principalmente para o manejo destinado ao controle integrado das doenças.

No Estado do Paraná três cultivares são as mais representativas: a) Burkett, com frutos arredondados, casca firme, amêndoas com coloração dourado a marrom-claro, sabor acentuado, tendendo para o amargo, com aproveitamento médio de $53 \%$ de amêndoa e $87,67 \mathrm{~kg} /$ árvore (DOVE, 2004). Muito susceptível à sarna (Cladosporium caryigenum) e "downy spot" (Mycosphaerella caryigena); b) Frotscher, frutos oblongo-elípticos, amêndoas grandes e atrativas. Boa resistência às duas doenças citadas e suscetível à "vein spot" (Gnomonia sp.) (GRAUKE \& THOMPSOM, 2004); c) Moneymaker, frutos oval-elípticos, amêndoas marrom e textura enrugada, é considerada precoce, produtiva e resistente à sarna (GRAUKE \& THOMPSOM, 2004).

Na pós-colheita, durante o armazenamento, as nozes estão sujeitas a alterações fisiológicas com perda da qualidade, principalmente por doenças de pós-colheita causadas por fungos que comprometem a qualidade principalmente pelo desenvolvimento de bolores. Entre esses, Penicillium spp., Cephalothecium roseum e
Aspergillus flavus, que além do emboloramento produzem aflotoxinas (ORTIZ \& CAMARGO, 2005). Também os fungos causadores de míldio podem afetar diretamente a casca das nozes, levando a amêndoa ao enrugamento. Poucos são os conhecimentos a respeito desses agentes em condições brasileiras.

Assim, o presente trabalho teve como objetivo determinar a incidência e identificação dos microrganismos associados à amêndoa e à casca de frutos de três cultivares de noz Pecan (Burkett, Frotscher e Moneymaker), produzidas na região norte do Estado do Paraná, após 30 dias de armazenamento à temperatura ambiente e submetidos e não à assepsia superficial.

Foram avaliadas, em condições de laboratório, nozes produzidas na safra de 2005 e colhidas aleatoriamente a uma altura de 2,0 m, nas árvores de noz Pecan selecionadas, pertencentes às cultivares Burkett, Frotscher e Moneymaker, em pomar localizado na Chácara Santa Rosa, Uraí-PR, latitude $23^{\circ} 18^{\prime} \mathrm{S}$ e longitude $50^{\circ} 64^{\prime} \mathrm{O}$, aos 190 dias após o florescimento.

Após colhidos, os frutos foram identificados, acondicionados em sacos de papel "kraft" e armazenados por trinta dias, em temperatura ambiente; procedeu-se à determinação da incidência dos microorganismos associados às cascas e às amêndoas. Para tanto, antes de serem seccionados ao meio para separação das cascas das amêndoas, os frutos foram submetidos à assepsia, tendo sido imersos em solução de álcool a 10\%, durante 2 minutos; depois, em solução de 3,0\% de hipoclorito, por cinco minutos; e, finalmente, lavados em água destilada esterilizada.

Em seguida, 100 frutos foram seccionados ao meio, no sentido longitudinal e as partes plaqueadas sobre três folhas de papel de filtro, umedecidas em água destilada e esterilizada, dispostas no interior de caixas plásticas tipo gerbox (11 x 11 x 3,5 cm), previamente desinfestadas com solução de hipoclorito a $1,05 \%$.

A incubação, durante sete dias, foi à temperatura de ambiente de laboratório, sob luz contínua (lâmpada fluorescente branca - tipo "luz do dia"). Após procedeuse à avaliação e à identificação dos microrganismos presentes com base em leituras individuais com auxílio de microscópio estereoscópico e óptico e também de literatura pertinente para confirmação dos resultados.

O delineamento experimental foi inteiramente casualizado, com 10 repetições, e cada parcela experimental constituída por 10 nozes, totalizando 100 sementes por tratamento. Foram calculadas as médias das parcelas e essas foram submetidas à análise descritiva. 
Nos frutos (cascas e amêndoas) das três cultivares foram encontrados associados em porcentuais variáveis, Alternaria sp.; Aspergillus ochraceus; A. flavus, A. niger, Cladosporium sp., Colletotrichum sp.; Fusarium sp.; Penicillium sp.; Rhizopus sp. e Bactéria spp. (Tabelas 1 e 2).

Para Cladosporium sp., a incidência foi de $100 \%$ em casca, com e sem assepsia; nas cultivares Burkett e Moneymaker e para Fusarium sp. foi detectado em porcentuais de $100 \%$ na amostra de amêndoa e casca, com e sem assepsia; para as cultivares Frotscher e Moneymaker apresentaram entre 80 e $90 \%$ de incidência nas cascas submetidas à assepsia superficial (Tabelas 1 e 2).
Nas amêndoas (parte comestível) e cascas, microrganismos importantes como Aspergillus flavus, A. niger e A. ochraceus; Cladosporium sp.; Fusarium sp. e Penicillium sp. foram os mais freqüentes (Tabelas 1 e 2). A adoção da assepsia com álcool a $10 \%$ e solução de $3,0 \%$ de hipoclorito não foi eficiente para eliminá-los, indicando que os mesmos já se associam às diferentes partes provavelmente antes da colheita, independente da cultivar. O Penicillium spp. e o A. flavus depreciam a qualidade das amêndoas por causa da formação de bolores e produção de toxina (ORTIZ \& CAMARGO, 2005). O Fusarium sp. além de produzir toxinas apodrece os frutos e sua presença pode significar perda de qualidade e valor para a comercialização.

Tabela 1 - Microrganismos associados à amêndoa de frutos de noz Pecan (Carya illinoensis), aos 30 dias do armazenamento e submetidos e não à assepsia superficial. Londrina,PR, 2007.

\begin{tabular}{|c|c|c|c|c|c|c|}
\hline \multirow{3}{*}{$\begin{array}{c}\text { Microrganismos } \\
\text { Associados }\end{array}$} & \multicolumn{6}{|c|}{ Incidência em \% nas amêndoas das cultivares } \\
\hline & \multicolumn{2}{|c|}{ Frotscher } & \multicolumn{2}{|c|}{ Burkett } & \multicolumn{2}{|c|}{ Moneymaker } \\
\hline & $\mathrm{C} / \mathrm{A}$ & S/A & $\mathrm{C} / \mathrm{A}$ & S/A & $\mathrm{C} / \mathrm{A}$ & S/A \\
\hline Alternaria sp. & 0 & 10 & 20 & 0 & 20 & 10 \\
\hline Aspergillus flavus & 70 & 70 & 0 & 50 & 0 & 20 \\
\hline Aspergillus niger & 20 & 80 & 0 & 0 & 0 & 0 \\
\hline Aspergillus ochraceus & 20 & 100 & 0 & 30 & 0 & 30 \\
\hline Cladosporium sp. & 20 & 20 & 50 & 90 & 50 & 100 \\
\hline Colletotrichum sp. & 0 & 0 & 30 & 10 & 30 & 0 \\
\hline Fusarium sp. & 100 & 100 & 100 & 100 & 100 & 100 \\
\hline Penicillium sp. & 100 & 100 & 30 & 70 & 30 & 90 \\
\hline Rhizopus sp. & 0 & 20 & 10 & 0 & 10 & 0 \\
\hline \multirow[t]{2}{*}{ Bactéria spp. } & 10 & 70 & 20 & 0 & 20 & 30 \\
\hline & 340 & 570 & 260 & 350 & 260 & 380 \\
\hline
\end{tabular}

C/A - com assepsia; S/A - sem assepsia.

Tabela 2 - Microrganismos associados à casca de frutos de noz Pecan (Carya illinoensis), aos 30 dias do armazenamento e, submetidos e não à assepsia superficial. Londrina,PR, 2007.

\begin{tabular}{lrrrrrr}
\hline \multirow{2}{*}{$\begin{array}{c}\text { Microrganismos } \\
\text { Associados }\end{array}$} & \multicolumn{2}{c}{ Frotscher } & \multicolumn{2}{c}{ Burkett } & \multicolumn{2}{c}{ Moneymaker } \\
\cline { 2 - 7 } & C/A & S/A & C/A & S/A & C/A & S/A \\
\hline Alternaria sp. & 80 & 100 & 90 & 100 & 100 & 80 \\
Aspergillus flavus & 10 & 10 & 0 & 0 & 0 & 30 \\
Aspergillus niger & 0 & 10 & 0 & 0 & 0 & 0 \\
Aspergillus ochraceus & 0 & 0 & 0 & 0 & 0 & 0 \\
Cladosporium sp. & 80 & 100 & 100 & 100 & 100 & 100 \\
Fusarium sp. & 80 & 100 & 100 & 100 & 90 & 100 \\
Penicillium sp. & 50 & 90 & 40 & 80 & 0 & 60 \\
Rhizopus sp. & 0 & 20 & 0 & 0 & 0 & 0 \\
Bactéria spp. & 0 & 0 & 30 & 40 & 0 & 0 \\
\hline & 300 & 430 & 360 & 420 & 290 & 370 \\
\hline
\end{tabular}

C/A - com assepsia; S/A - sem assepsia. 
Fungos importantes, como formadores de bolores e produtores de micotoxinas em alimentos, podem se desenvolver e estar presentes externa e internamente em castanhas de noz Pecan das cultivares Burkett, Frotscher e Moneymaker.

Os resultados obtidos mostram que a boa qualidade sanitária de amêndoas de noz Pecan é obtida através de práticas e manejos que reduzam a incidência dos microrganismos a partir dos estágios que vão do florescimento à colheita; depois, muito pouco pode ser feito para a garantia do produto. Tratamentos e assepsias não garantem a boa qualidade.

\section{REFERÊNCIAS BIBLIOGRÁFICAS}

BALL, J. The tasty pecan. Washington, DC: Amerian Forests, 2004. Disponível em: <http:// www.americanforests.org/productsandpubs/magazine/ archives/2001 autumn/inprofile.php>. Acesso em: 20 maio 2004.

DOVE, S. K. Average nut quality characteristics. Georgia: Horticulture Departament of University of Georgia, 2004. Disponível em: <http://www.cpes.peachnet.edu/pecan/ table1.html>. Acesso em: 20 out. 2004.
GRAUKE, L. J.; THOMPSOM, T. E. Pecan cultivars. Pecan Breeding \& Genetics, Somerville, 2004. Disponível em: $<$ http://extension-horticulture.tamu.edu/carya/pecans/ pecalph.htm>. Acesso em: 21 out. 2004.

INSTITUTE FOR TROPICAL AND SUBTROPICAL CROPS (África do Sul). Cultivating pecan nuts. Pretoria: National Department of Agriculture, 2004. Disponível em: <http://www.nda.agric.za/docs/pecan/pecan.htm>. Acesso em: 16 ago. 2004.

ORTIZ, E. R. N.; CAMARGO, L. E. A. Doenças da Nogueira Pecan. In: KIMATI, H.; AMORIM, L.; BERGAMIN FILHO, A.; CAMARGO, L. E. A.; REZENDE, J. A. M. (Eds.). Manual de fitopatologia: doenças das plantas cultivadas. 4. ed. São Paulo: Agronômica Ceres, 2005. cap. 57, p. 501-505.

REIGER, M. Pecan - Carya illinoensis (Wangenh.) K. Koch. Georgia: University of Georgia, 2004. Disponível em: <http:/ /www.uga.edu/fruit\%20/pecan.htm>. Acesso em: 21 jun. 2004.

SIMÃO, S. Nogueira pecã. In: Tratado de fruticultura. Piracicaba: FEALQ, 1998. cap. 14, p. 635-649. 